\title{
Breast Cancer Detection in Qatar: Evaluation of Mammography Image Quality Using A Standardized Assessment Tool
}

\author{
Anand K. Narayan ${ }^{1}$ (D), Huda Al-Naemi ${ }^{2}$ (D), Antar Aly ${ }^{2}$ (D), Mohammad Hassan Kharita ${ }^{2}$ (D), Ruhani Doda Khera ${ }^{1}$ (D), \\ Mohamad Hajaj $^{2}$ (D), Madan M. Rehani ${ }^{1}$ \\ ${ }^{1}$ Massachusetts General Hospital, Boston, MA, USA \\ ${ }^{2}$ Hamad Medical Corporation, Doha, Qatar
}

\begin{abstract}
Objective: Compared with other countries in the Middle East, Qatar has one of the highest breast cancer incidence and mortality rates. Poor quality mammography images may be associated with advanced stage breast cancer, however there is limited information about the quality of breast imaging in Qatar. Our purpose was to evaluate the clinical image quality of mammography examinations performed at a tertiary care center in Doha, Qatar using a standardized assessment tool.

Materials and Methods: Bilateral mammograms from consecutive patients from a tertiary care cancer center in Doha, Qatar were obtained. Proportions of examinations deemed adequate for interpretation were estimated. Standardized clinical image quality assessment form was utilized to evaluate image quality components. For each image, image quality components were given grades on a 1-5 scale (5- excellent, 4- good, 3- average, 2- fair, 1- poor). Mean scores with $95 \%$ confidence intervals were estimated for each component.

Results: Consecutive sample of 132 patients was obtained representing 528 mammographic images. Overall, $99.2 \%$ of patients underwent examinations rated as acceptable for interpretation. Mean scores for each image quality component ranged from 4.045 to 5.000 (lowest score for inframammary fold). Image quality component scores were $93.0 \%$ excellent, $5.2 \%$ good, $1.1 \%$ average, $0.6 \%$ fair, and $0.1 \%$ poor.

Conclusion: Overall image quality at a tertiary care center in Doha, Qatar was acceptable for interpretation with minimal areas identified for improvement.
\end{abstract}

Keywords: Breast cancer, diagnostic imaging, Qatar, mammography, Middle East

Cite this articles as: Narayan A, Al-Naemi H, Aly A, Kharita MH, Khera RD, Hajaj M, et al. Breast Cancer Detection in Qatar: Evaluation of Mammography Image Quality Using A Standardized Assessment Tool. Eur J Breast Health 2020; 16(2): 124-128.

\section{Introduction}

Compared with other countries in the Middle East, Qatar has one of the highest breast cancer incidence and mortality rates (1). In a retrospective review of 268 breast cancer patients in Chicago, Rauscher et al. found that lower image quality scores were associated with late stage breast cancer diagnoses, even after adjusting for patient and practice related characteristics (2). There is limited information about mammography image quality in Qatar. Our purpose was to evaluate the clinical image quality of mammography examinations performed at a tertiary care center in Doha, Qatar using a standardized assessment tool.

\section{Materials and Methods}

Ethics committee and institutional review board (IRB) approval was obtained for our retrospective study. Informed consent was not required for retrospective review of previously collected images. This study was supported by a grant from the Qatar National Research Fund (QNRF), project number NPRP9-189-3-031. 
Study results were reported using Strengthening the Reporting of Observational Studies in Epidemiology (STROBE) guidelines for reporting observational studies (3).

\section{Study design}

Our study design involved retrospective evaluation of consecutive images performed from October 2018 to January 2019.

\section{Data sources/measurement}

Clinical image quality of mammography images was evaluated using Hologic SecureView Dx viewing software (Hologic Inc., Marlborough, MA, United States). Images were acquired using Hologic Selenia Dimensions 2D and 3D mammography units installed in 2016 (Hologic Inc., Marlborough, MA, United States). Initial evaluation of clinical image quality was performed by the interpreting radiologist. External review of clinical image quality review was subsequently performed by a breast imaging faculty member with 4 years of experience in breast imaging. Review was blinded to final assessment, whether or not examination was technically recalled, and the technologist performing the examination.

\section{Setting}

Mammography images were acquired from two sites associated with a tertiary care center in Doha, Qatar. The tertiary care center is the main provider of secondary and tertiary healthcare in Qatar (4). At both sites, women present for diagnostic mammography.

\section{Participants}

Images from consecutive patients from two different imaging sites was obtained from October 2018 to January 2019 was included.

\section{Study size}

The study size was based on available number of eligible adult patients presenting to the two sites.

\section{Variables}

\section{Dependent variables}

Outcomes for the study included subjective binary assessment as to whether or not the examination was technically adequate for clinical interpretation (Adequate vs Not Adequate for Interpretation) as well as outcomes from standardized assessment form. Standardized image quality assessment was derived from recommendations produced by a working group of the National Health Service Breast Screening Programme (NHSBSP) Clinical and Professional group for Radiography, developed for the National Health Service in the UK (5). The standard image quality assessment form involved subjective assessment of the following items (Figure 1): Correct patient ID \& Markers, Appropriate

\section{Key Points}

- $99 \%$ of studies conducted at a tertiary care cancer center in Doha, Qatar were deemed adequate for interpretation, results that are comparable to academic centers in the United States and Europe.

- Using our standardized quality improvement instrument, we found that visualization of the inframammary fold was the most commonly identified area for improvement.

- Standardization of performance and evaluation of mammography images will be essential to maintaining and improving the quality of screening and diagnostic breast imaging. exposure, Adequate compression to hold breast firmly - no movement, Image sharp, No artefacts obscuring image, No obscuring skin folds, Nipple in profile, Pectoral muscle to nipple level, Pectoral muscle at appropriate angle, IMF shown clearly, Medial border demonstrated, Back of breast clearly shown with some medial central \& lateral, Some axillary tail shown. Appropriate exposure refers to subjective reader perception of exposure (standardized quality assessment form does not include quantitative benchmarks or reference levels for radiation dose). For each item, each image (RMLO, LMLO, RCC, LCC) was given a 1-5 score (1- poor, 2- fair, 3- average, 4- good, 5- excellent). For patients who had repeat views, each image quality component was evaluated on the best possible view obtained for the evaluation of each specific component of image quality.

\section{Independent variables}

Independent variables included imaging site, age and breast density (categorized as dense ("The breasts are heterogeneously dense, which may obscure small masses", "The breasts are extremely dense, which lowers the sensitivity of mammography") vs not dense ("The breasts are almost entirely fatty", "There are scattered areas of fibroglandular density").

\section{Statistical analysis}

Proportion of examinations considered as adequate were estimated, stratified by imaging site and breast density. For each component of clinical image quality for each image, means and 95\% confidence intervals were estimated. Multiple variable linear regression analyses were conducted to evaluate the association between image quality parameters and imaging site, age and breast density. Analyses were conducted using STATA 11 (StataCorp, College Station, TX, United States). Two-tailed $\mathrm{p}$ values less than 0.05 were considered statistically significant.

\section{Results}

A total of 528 images were obtained from 2 sites from 132 unique patients (90 patients from one site and 42 patients from the other site). All of the examinations $(100 \%)$ in our study were initially interpreted as technically adequate by the interpreting radiologist. External review of these examinations found $99.2 \%$ (131/132) of patients undergoing technically adequate examinations for clinical interpretation. The one discrepant examination was one in which image quality was considered as not adequate for interpretation was rated as inadequate secondary to the sharpness of the LMLO image (rated as poor in the standardized image quality assessment form).

Quantitative ratings for each of the image quality components from external review are presented in Table 1 with $95 \%$ confidence intervals. Mean scores for each image quality component ranged from 4.045 to 5.000 with a few of the indicators having perfect image quality scores (Correct Patient ID, Medial border demonstrated, Back of breast clearly shown with some medial central \& lateral, Some axillary tail shown). Overall $93.0 \%$ of the image quality component scores were excellent $(5,404 / 5,808), 5.2 \%$ were good, $(304 / 5,808), 1.1 \%$ were average $(66 / 5,808), 0.6 \%$ were fair $(33 / 5,808)$ and $0.1 \%$ were poor $(1 / 5,808)$. Image quality component with the lowest score was inframammary fold (mean 4.083) with $40.9 \%$ of the images with excellent scores, $35.6 \%$ of the images with good scores, $14.4 \%$ of the images with average scores, $9.1 \%$ of the images with fair scores and $0.0 \%$ of the images with poor scores. 


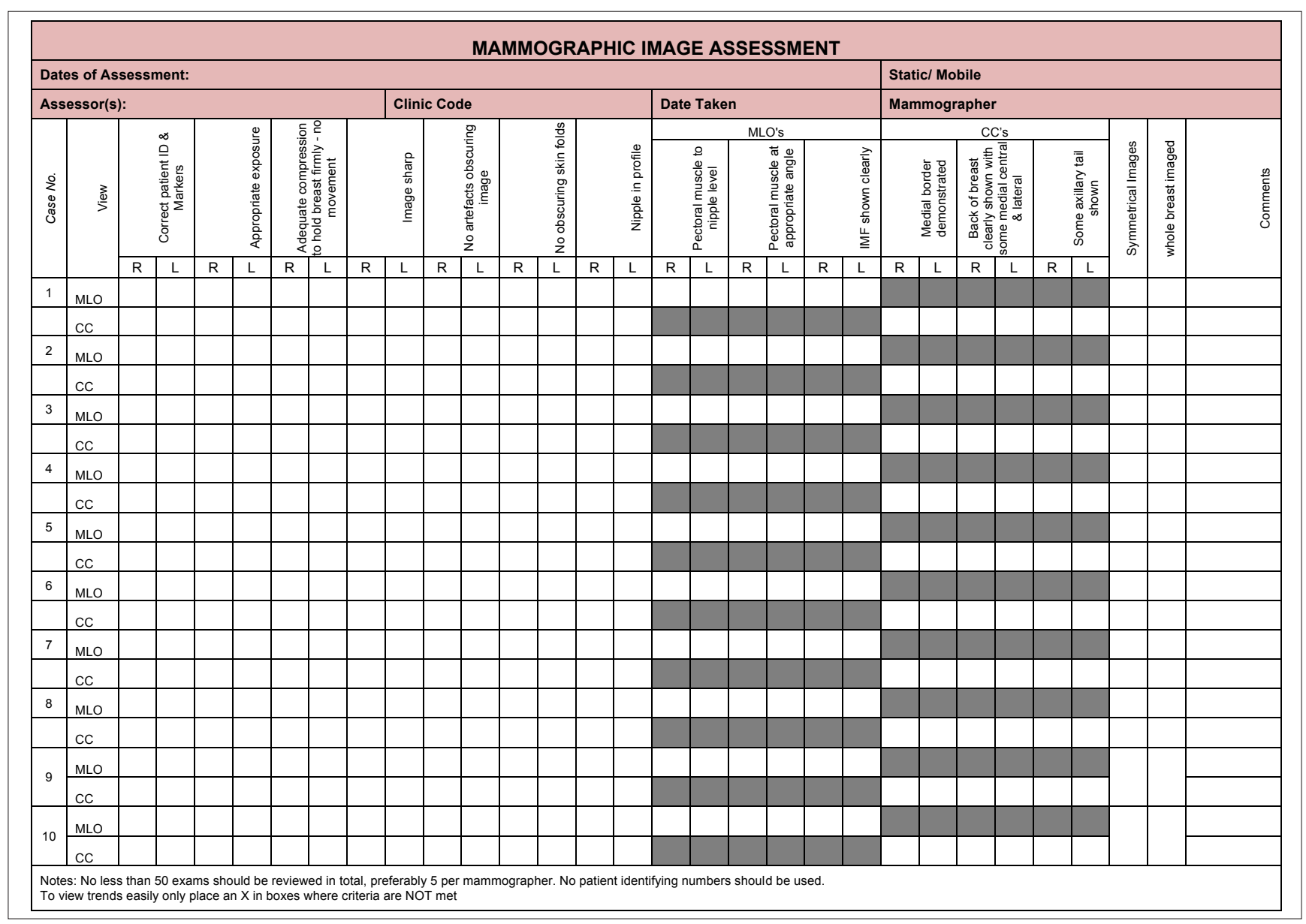

Figure 1. Standardized image quality assessment form

\section{Discussion and Conclusion}

In our study, 99\% of studies conducted at a tertiary care center in Doha, Qatar were deemed adequate for interpretation, results that are comparable to high volume academic centers in the United States and Europe $(6,7)$. With adequate image quality performance, our results suggest that image quality may not be an explanation for higher rates of advanced stage breast cancer in Qatar.

As the country develops additional capacity and awareness for mammography screening, it will be important to continuously monitor image quality. Using our standardized instrument, we identified a few specific areas for improvement, specifically the inframammary fold. NHS Breast Screening Guidance for breast screening mammographers states that the inframammary angle should be clearly demonstrated without overlapping chest wall tissue. This positioning ensures that the breast has been lifted and that the postero-inferior part of the breast has been adequately imaged. Previously published studies have suggested that the inframammary fold is one of the most commonly cited reasons for technical recalls (8). Writing in the Society of Breast Imaging Newsletter, Louise C. Miller provided several suggestions for improving visualization of the IMF and reducing skin folds (9). To improve visualization of the IMF, the IMF should be placed onto the image receptor. To reduce folds, technologists may utilize several corrective actions including smoothing lateral and inferior breast tissue before lifting the breast up and out, maintaining the up and out position throughout breast compression, and having the patient lift her standardized instrument can help facilitate comprehensive longitudinal monitoring of components of clinical image quality.

High performance quality control for breast images includes not only evaluation of image quality of clinical images but also breast phantoms. Phantoms quantitatively evaluate the capacity of mammographic systems to image structures, similar to those found clinically. Gürdemir and Aribal used the American College of Radiology (ACR) phantom to assess the image quality of mammography units in Istanbul (10). The ACR phantom contains 16 objects that mimic structures seen clinically (spiculations, microcalcifications and small masses). Images from $38 \%$ of the imaging units had unacceptable quality scores. In our study, we found that $99 \%$ of studies had acceptable image quality study, however our study focused on clinical image quality evaluation. Future studies should be conducted including breast phantoms to evaluate whether or not imaging units in Qatar can identify structures that mimic early breast cancers.

Standardization of performance and evaluation of mammography images has been associated with improvements in image quality (6). The International Atomic Energy Agency (IAEA) convened a group of breast imagers, medical physicists and radiographers to evaluate and improve image quality in 15 countries (Bosnia and Herzegovina, Costa Rica, Egypt, India, Kenya, the Frmr. Yug. Rep. of Macedonia, Mexico, Nigeria, Pakistan, Philippines, Slovenia, Turkey, Uganda, United Kingdom and Zambia) $(11,12)$. After performing a baseline evaluation in these countries, the IAEA recommended several key interventions to foster high quality imaging practices. Among these interven- 
Table 1. Quantitative evaluation of image quality components

\begin{tabular}{lcccc} 
Parameter & RMLO* & LMLO* & RCC* & LCC* \\
\hline Correct Patient ID & $5(5,5)$ & $5(5,5)$ & $5(5,5)$ & $5(5,5)$ \\
Exposure & $4.992(4.977,5.007)$ & $5(5,5)$ & $4.992(4.977,5.007)$ & $5(5,5)$ \\
Compression & $4.970(4.940,4.999)$ & $4.970(4.933,5.006)$ & $4.992(4.977,5.007)$ & $5(5,5)$ \\
Sharpness & $4.902(4.846,4.957)$ & $4.833(4.745,4.921)$ & $4.939(4.888,4.990)$ & $4.788(4.717,4.859)$ \\
Artefacts & $4.985(4.964,5.006)$ & $4.992(4.977,5.007)$ & $5(5,5)$ & $5(5,5)$ \\
Skin Folds & $4.758(4.684,4.832)$ & $4.742(4.664,4.821)$ & $4.977(4.952,5.003)$ & $4.795(4.726,4.865)$ \\
Nipple & $4.879(4.794,4.964)$ & $4.894(4.806,4.982)$ & $4.909(4.840,4.978)$ & $4.856(4.758,4.954)$ \\
Pectoralis & $4.841(4.762,4.920)$ & $4.879(4.815,4.943)$ & & $5(5,5)$ \\
Pectoralis Angle & $4.917(4.860,4.973)$ & $4.955(4.913,4.996)$ & & $5(5,5)$ \\
IMF* & $4.045(3.874,4.217)$ & $4.121(3.963,4.279)$ & & $5(5,5)$ \\
Back of Breast Shown & & & $5(5,5)$ & $5(5,5)$ \\
Axillary Tail Shown & & & $4.992(4.977,5.007)$ \\
Medial border & & & & \\
\hline LCC: left craniocaudal; LMLO: left mediolateral oblique; RCC: right craniocaudal; RMLO: right mediolateral oblique &
\end{tabular}

tions, the IAEA recommended additional training in mammography positioning for technologists and performance measurements and dosimetry for medical physicists. Implementation of these recommendations and several others were associated with improved breast imaging quality, capability and expertise. The improvements were particularly pronounced in diagnostic imaging centers, similar to the two sites associated with the tertiary care center where our study was performed. While our study focused on radiography, these types of multi-pronged, collaborative initiatives will be essential to maintaining and improving the quality of screening and diagnostic breast imaging.

Limitations of our study include image quality evaluation performed by one reader and selection of study participants from two sites associated with one center. Our study was limited as external image quality evaluation was performed by just one reader. Though readings by multiple readers would provide more optimal evaluation of image quality parameters, results from the overall readings by one reader were similar to previously published evaluations of clinical image quality, suggesting that double reading may not have significantly changed overall conclusions. Our study was also limited by the utilization of two sites associated with one center. While there are other sites that perform mammography screening, our study site is the site that provides tertiary cancer care for patients in Qatar. Finally, our study was performed at diagnostic mammography sites. To develop a comprehensive picture of overall image quality, our study would also need to include screening sites. Unfortunately, implementation of breast cancer screening in Qatar has been limited (13). While widespread implementation of mammographic screening plays a critical role in reducing breast cancer mortality, expanding access to diagnostic mammography may facilitate access to high quality, effective treatment in women with later stage breast cancers (14).

In conclusion, 99\% of studies conducted at a tertiary care cancer center in Doha, Qatar were deemed adequate for interpretation, results that are comparable to high performance academic centers in the United States and Europe.
Ethics Committee Approval: Ethics committee approval was received for this study from the ethics committee of Hamad Medical Corporation (project number NPRP9-189-3-031).

Informed Consent: Informed consent was not required for retrospective review of previously collected images.

Peer-review: Externally peer-reviewed.

Author Contributions: Concept - A.K.N., M.M.R., H.A.K.N.; Design - A.K.N., M.M.R., A.A.; Supervision - A.K.N., A.A., M.H., H.A.K.N., M.M.R., M.H.K.; Resources - M.H.K., A.A., M.M.R., H.A.K.N.; Materials - M.M.R., H.A.K.N., A.A., M.H.K., M.H.; Data Collection and/or Processing - R.D.K., A.K.N., M.M.R., A.A., M.H.; Analysis and/or Interpretation - A.K.N., R.D.K., M.M.R., A.A.; Literature Search - A.K.N., M.M.R., A.A., M.H., R.D.K.; Writing Manuscript - A.K.N.; Critical Review - A.K.N., R.D.K., M.M.R., A.A., M.H., M.H.K., H.A.K.N.

Conflict of Interest: The authors have no conflicts of interest to declare.

Financial Disclosure: This study was supported by a grant from the Qatar National Research Fund (QNRF), project number NPRP9-1893-031.

\section{References}

1. Chouchane L, Boussen H, Sastry KS. Breast cancer in Arab populations: molecular characteristics and disease management implications. Lancet Oncol 2013; 14: e417-424. (PMID: 23993386) [CrossRef]

2. Rauscher GH, Conant EF, Khan JA, Berbaum ML. Mammogram image quality as a potential contributor to disparities in breast cancer stage at diagnosis: an observational study. BMC Cancer 2013; 13: 208. (PMID: 23621946) [CrossRef]

3. Vandenbroucke JP, von Elm E, Altman DG, Gøtzsche PC, Mulrow CD, Pocock SJ, et al. Strengthening the Reporting of Observational Studies in Epidemiology (STROBE): explanation and elaboration. Epidemiology 2007; 18: 805-835. (PMID: 18049195) [CrossRef]

4. Mohsen H, Haddad P, Allam A, Hassan A. Patterns in place of cancer death in the State of Qatar: a population-based study. PLoS One 2014; 9: e109615. (PMID: 25536076) [CrossRef] 
5. NHS Breast Screening Programme. Guidance for breast screening mammographers. Public Health England (serial online) 2006 Apr: Available from: URL: https://www.gov.uk/government/publications/breast-screening-quality-assurance-for-mammography-and-radiography

6. Bassett LW, Hirbawi IA, DeBruhl N, Hayes MK. Mammographic positioning: evaluation from the view box. Radiology 1993; 188: 803-806. (PMID: 8351351) [CrossRef]

7. NHS Breast Screening Programme. Guidance on collecting, monitoring and reporting technical recall and repeat examinations. Public Health England (serial online) 2006 Nov: Available from: URL: https:/www. gov.uk/government/publications/breast-screening-repeat-mammograms

8. Huppe AI, Overman KL, Gatewood JB, Hill JD, Miller LC, Inciardi MF. Mammography positioning standards in the digital era: is the status quo acceptable? AJR Am J Roentgenol 2017; 209: 1419-1425. [Epub ahead of print]. (PMID: 28871810) [CrossRef]

9. Miller LC. Common problems with the mediolateral oblique: how to help your technologist. the member newsletter of the society of breast imaging. 2016. Available from: https://www.mammographyeducation.com/ wp-content/uploads/2014/09/SBI_Newsletter_Issue4_2016_CommonProblems-with-the-Mediolateral-Oblique-How-to-Help-Your-Technologist.pdf. (Accessed 12/28/2019)
10. Gürdemir B, Arıbal E. Assessment of mammography quality in Istanbul. Diagn Interv Radiol 2012; 18: 468-472. (PMID: 22801869) [CrossRef]

11. Mora P, Faulkner K, Mahmoud AM, Gershan V, Kausik A, Zdesar U, et al. Improvement of early detection of breast cancer through collaborative multi-country efforts: medical physics component. Phys Med 2018; 48: 127-134. (PMID: 29599081) [CrossRef]

12. Aribal E, Mora P, Chaturvedi AK, Hertl K, Davidović J, Salama DH, et al. Improvement of early detection of breast cancer through collaborative multi-country efforts: observational clinical study. Eur J Radiol 2019; 115: 31-38. (PMID: 31084756) [CrossRef]

13. Donnelly TT, Khater AH, Al-Bader SB, Al Kuwari MG, Malik M, AlMeer N, et al. Factors that influence awareness of breast cancer screening among Arab women in Qatar: results from a cross sectional survey. Asian Pac J Cancer Prev 2014; 15: 10157-10164. (PMID: 25556441) [CrossRef]

14. Howard DH, Ekwueme DU, Gardner JG, Tangka FK, Li C, Miller JW. The impact of a national program to provide free mammograms to lowincome, uninsured women on breast cancer mortality rates. Cancer 2010; 116: 4456-4462. (PMID: 20564744) [CrossRef] 